13

\title{
Влияние усиленной электрическим полем термической электронной эмиссии на температуру катода с тонкой диэлектрической пленкой в дуговом газовом разряде
}

\author{
(C) Г.Г. Бондаренко, ${ }^{1}$ М.С. Дубинина, ${ }^{2}$ В.И. Кристя ${ }^{2}$ \\ ${ }^{1}$ Национальный исследовательский университет „Высшая школа экономики“, \\ 101000 Москва, Россия \\ ${ }^{2}$ Московский государственный технический университет им. Н.Э. Баумана, Калужский филиал, \\ 248000 Калуга, Россия \\ e-mail: kristya@bmstu-kaluga.ru
}

Поступило в Редакцию 19 ноября 2018 г.

В окончательной редакции 5 ноября 2019 г.

Принято к публикации 5 ноября 2019 г.

\begin{abstract}
Предложена модель усиленной электрическим полем термической (термополевой) эмиссии электронов из металлической подложки катода в тонкую диэлектрическую пленку на его поверхности. Сформулирована система уравнений для температуры поверхности катода в дуговом разряде и напряженности электрического поля в пленке, обеспечивающих необходимую плотность разрядного тока. Показано, что наличие диэлектрической пленки может приводить к существенному понижению температуры катода в разряде вследствие меньшей высоты потенциального барьера на границе металл-диэлектрик, чем на границе металлразряд при ее отсутствии. Установлено, что вследствие усиления термической эмиссии электронов в пленку возникающим в ней электрическим полем происходит дополнительное снижение температуры катода на величину порядка $100 \mathrm{~K}$.
\end{abstract}

Ключевые слова: диэлектрическая пленка на катоде, термополевая электронная эмиссия, эмиссионная эффективность пленки, температура катода.

DOI: 10.21883/JTF.2020.05.49191.403-18

\section{Введение}

В дуговых газоразрядных приборах, таких как осветительные лампы, после подачи напряжения на электроды сначала зажигается тлеющий разряд, в котором основным механизмом эмиссии с катода электронов, необходимых для поддержания разряда, является ионноэлектронная эмиссия. Через некоторое время температура катода в результате его нагрева потоком тепла, поступающего из разряда, достигает значений, при которых возможна термическая электронная эмиссия, и разряд переходит в дуговую форму [1-5]. Если у нагретого катода существует достаточно сильное электрическое поле, с него может происходить усиленная полем термическая (термополевая) эмиссия электронов [6,7]. Она обеспечивает возникновение дугового разряда при более низкой температуре катода, чем термическая эмиссия, что должно приводить к снижению интенсивности испарения материала катода в нем и к увеличению срока службы прибора.

В газовых разрядах с металлическим катодом (а также в вакууме при наличии на катоде тонкой оксидной пленки) термополевая эмиссия возможна лишь в случае, если напряженность электрического поля у его поверхности превышает $10^{8} \mathrm{Vm}^{-1}$ [7-10], что может иметь место только при давлении рабочего газа на 2-3 порядка большем атмосферного [11], а при его давлении по- рядка атмосферного - в микронных межэлектродных промежутках (в микроразрядах) [12,13]. В условиях же, характерных для газоразрядных ламп (давление рабочего газа до $10^{6} \mathrm{~Pa}$ и межэлектродное расстояние, превышающее $10^{-3} \mathrm{~m}$ ), напряженность электрического поля в разряде недостаточно велика, и термополевой механизм электронной эмиссии не играет заметной роли $[6,7,14,15]$.

Одним из факторов, облегчающих переход тлеющего разряда в дуговой, является наличие на катоде тонких диэлектрических включений [16-19]. Поэтому в состав электродов дуговых ламп для улучшения эмиссионных свойств обычно добавляют оксиды металлов, такие как $\mathrm{BaO}, \mathrm{MgO}, \mathrm{Al}_{2} \mathrm{O}_{3}$, являющиеся диэлектриками [20-22]. При нагреве на их поверхности могут формироваться тонкие диэлектрические оксидные пленки толщиной $10^{1}-10^{2} \mathrm{~nm}$, испарение вещества которых в разряде компенсируется его поступлением из объема электрода в результате диффузии. В результате при протекании разрядного тока на электроде, выполняющем функции катода, происходит накопление положительных зарядов $[23,24]$, приводящее к возникновению в пленке электрического поля с напряженностью, достаточной для возникновения полевой эмиссии в нее электронов из металлической подложки. Такие электроны ускоряются в пленке полем и, достигая ее внешней границы, нейтрализуют поверхностный заряд, в результате чего устанав- 
ливается стационарный режим разряда. Некоторая доля электронов, величина которой называется эмиссионной эффективностью пленки [25,26], может преодолевать потенциальный барьер на границе пленки и выходить в разрядный объем, создавая эмиссионный ток. В процессе нагрева такого катода в разряде полевая электронная эмиссия из его подложки в диэлектрическую пленку вследствие наличия в ней сильного поля должна переходить в термополевую при произвольных величинах межэлектродного расстояния и давления рабочего газа в приборе. Однако роль усиленной полем термической электронной эмиссии в процессе горения дугового разряда при наличии на катоде диэлектрической пленки до настоящего времени не изучена.

В настоящей работе сформулирована модель, описывающая усиленную электрическим полем термическую эмиссию электронов из металлической подложки электрода в диэлектрическую пленку, а также их движение в пленке и выход в разрядный объем. Получено выражение для эмиссионной эффективности пленки и оценено влияние такого механизма эмиссии на температуру катода в дуговом разряде.

\section{Описание модели}

Пусть на металлическом катоде газоразрядного прибора, нагретом до достаточно высокой температуры $T$, находится тонкая диэлектрическая пленка толщиной $H_{f}$. При его бомбардировке ионами в разряде на внешней поверхности пленки накапливается положительный заряд, что при $H_{f} \approx 10 \mathrm{~nm}$ приводит к возникновению в ней однородного электрического поля с напряженностью $E_{f}[27,28]$. Будем считать, что координата $z$ направлена перпендикулярно поверхности катода, причем граница металлической подложки катода и пленки находится в плоскости $z=0$, а внешняя граница пленки совпадает с плоскостью $z=H_{f}$. Тогда при учете силы изображения потенциальная энергия электрона в диэлектрике, отсчитываемая от дна зоны проводимости металла, определяется выражением $[6,7]$

$$
V(z)=\varepsilon_{F}+\varphi_{m}-\chi_{d}-e \varphi(z),
$$

где $\varphi(z)=E_{j} z+k e / 4 z-$ потенциал электрического поля в пленке, $k=1 / 4 \pi \varepsilon_{0} \varepsilon_{f}, \varepsilon_{f}$ и $\varphi_{m}$ - энергия Ферми и работа выхода подложки, $\chi_{d}$ и $\varepsilon_{f}$ - электронное сродство и высокочастотная диэлектрическая проницаемость материала пленки, $e$ - величина заряда электрона, $\varepsilon_{0}$ - диэлектрическая постоянная. Энергетическая диаграмма системы металл-диэлектрик-разряд изображена на рис. 1.

Когда напряженность электрического поля в пленке не превосходит $10^{8} \mathrm{Vm}^{-1}$, в дуговом разряде происходит термическая эмиссия электронов над потенциальным барьером (траектория 1 на рис. 1), максимальная высота которого $\varepsilon_{m}=\varepsilon_{F}+\varphi_{m}-\chi_{d}-\sqrt{k e^{3} E_{f}}$ достигается

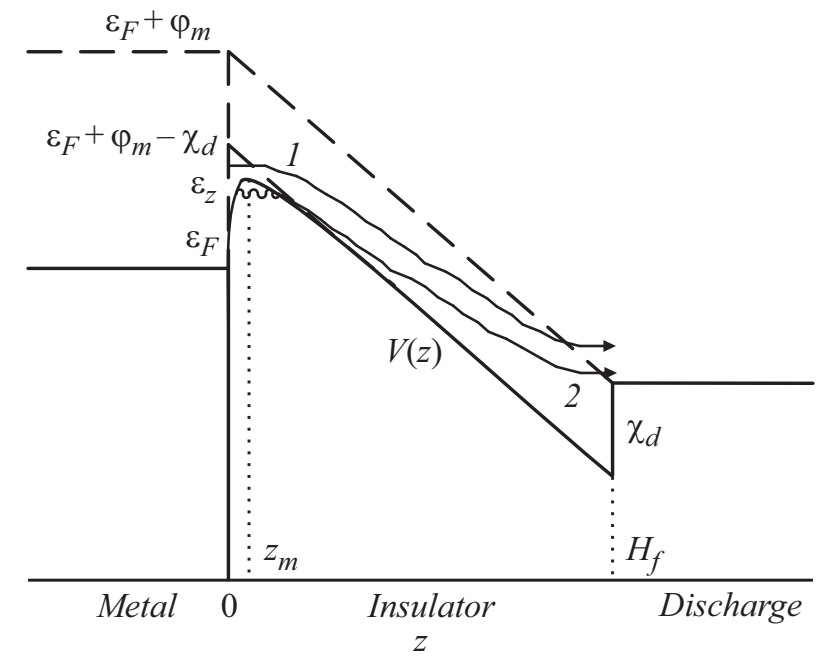

Рис. 1. Энергетическая диаграмма системы металлдиэлектрик-разряд.

в точке $z_{m}=\sqrt{k e / 4 E_{f}} \ll H_{f}$. При увеличении поверхностного заряда на пленке напряженность электрического поля $E_{f}$ в ней возрастает, ширина потенциального барьера уменьшается, и становится возможным туннелирование электронов через барьер вблизи его вершины (траектория 2 на рис. 1), т. е. возникает усиленная полем термическая эмиссия электронов из металла в зону проводимости диэлектрика.

Функция распределения эмитируемых в пленку электронов по продольной компоненте энергии $\varepsilon_{z}$, с учетом того что для них $\varepsilon_{z}-\varepsilon_{F} \gg k_{b} T$, при этом имеет вид $[6,7,29]$ :

$$
\begin{aligned}
f_{t f}\left(\varepsilon_{z}\right)= & \frac{4 \pi m_{e}^{*} k_{b} T}{h^{3}} \exp \left(-\frac{\varepsilon_{z}-\varepsilon_{F}}{k_{b} T}\right) \\
& \times\left[1+\exp \left(\pi a \frac{(1-y)}{y}\right)\right]^{-1},
\end{aligned}
$$

а плотность их тока равна

$$
j_{t f}=e \int_{0}^{\infty} f_{t f}\left(\varepsilon_{z}\right) d \varepsilon_{z},
$$

где $\quad a=\left(16 \pi^{4} k^{3} e^{5} m^{* 2} / h^{4} E_{f}\right)^{1 / 4}, \quad y=\sqrt{k e^{3} E_{f}} /\left(\varepsilon_{F}+\right.$ $\left.+\varphi_{m}-\chi_{d}-\varepsilon_{z}\right), m_{e}^{*}-$ эффективная масса электрона в диэлектрике, $k_{b}$ - постоянная Больцмана, $h$ постоянная Планка.

Подстановка в (3) соотношения (2) при условии, что электрическое поле в пленке не очень сильное, так что выполняется условие $h_{0}=h_{0}\left(T, E_{f}\right)=$ $=\sqrt{k e^{3} E_{f}} / \pi a k_{b} T<1$, дает после вычисления интеграла выражение для плотности тока усиленной полем 
термической электронной эмиссии $[6,7,30]$ :

$f_{t f}=e \frac{4 \pi m^{*}\left(k_{b} T\right)^{2}}{h^{3}} \exp \left(-\frac{e\left(\varphi_{m}-\chi_{d}\right)-\sqrt{k e^{3} E_{f}}}{k_{b} T}\right) g\left(h_{0}\right)$,

где множитель $g\left(h_{0}\right)=\pi h_{0} / \sin \left(\pi h_{0}\right)$ учитывает влияние туннелирования электронов через барьер на величину плотности тока электронной эмиссии из подложки катода в пленку.

Эмитируемые в зону проводимости пленки электроны ускоряются электрическим полем в направлении ее внешней границы и тормозятся при рассеянии на фононах [25,26,31]. Функция распределения их потока по $\varepsilon_{z}$ в пленке может быть найдена путем решения соответствующего одномерного кинетического уравнения [26] и у ее внешней границы, т.е. при $z=H_{f}$, определяется выражением

$$
\begin{aligned}
& f_{e}\left(H_{f}, \varepsilon_{z}\right)=\sum_{n=0}^{\infty} f_{e n}\left(H_{f}, \varepsilon_{z}\right)=\exp \left(-H_{f} / \lambda_{e}\right) \\
& \times \sum_{n=0}^{\infty}\left(\left(H_{f} / \lambda_{e}\right)^{n} / n !\right) f_{t f}\left(\varepsilon_{z}+n \Delta \varepsilon-e \varphi\left(H_{f}\right)\right),
\end{aligned}
$$

где $f_{e n}\left(H_{f}, \varepsilon_{z}\right)$ - энергетическое распределение электронов, претерпевших в пленке $n$ столкновений с фононами до точки с координатой $z=H_{f}, \Delta \varepsilon-$ энергия, теряемая электроном при каждом столкновении с фононом, $\lambda_{e}-$ средняя длина пробега электрона вдоль оси $z$ между столкновениями.

Интегрирование (5) по $\varepsilon_{z}$ дает

$$
j_{t}\left(H_{f}\right)=e \int_{0}^{\infty} f_{e}\left(H_{f}, \varepsilon_{z}\right) d \varepsilon_{z}=j_{t f},
$$

так как в стационарном режиме разряда плотности тока эмитированных электронов в пленке у ее внутренней и внешней границ должны совпадать.

Плотность же тока электронов, выходящих из пленки в разрядный объем, равна [26]

$$
\begin{aligned}
j_{e}\left(H_{f}\right) & =e \int_{\varepsilon_{m}}^{\infty} f_{e}\left(H_{f}, \varepsilon_{z}\right) d \varepsilon_{z} \\
& =\exp \left(-H_{f} / \lambda_{e}\right) \sum_{n=0}^{\infty}\left(\left(H_{f} / \lambda_{e}\right)^{n} / n !\right) j_{e n}
\end{aligned}
$$

причем

$$
j_{e n}=e \int_{\varepsilon_{m}}^{\infty} f_{t f}\left(\varepsilon_{z}+n \Delta \varepsilon-e \varphi\left(H_{f}\right)\right) d \varepsilon_{z},
$$

где $\varepsilon_{m}=\varepsilon_{F}+\varphi_{m}$.

Соотношение (8) с учетом (2) может быть приведено к виду

$$
j_{e n}=e \frac{4 \pi m^{*}\left(k_{b} T\right)^{2}}{h^{3}} \exp \left(-\frac{\varphi_{m}-\chi_{d}-\sqrt{k e^{3} E_{f}}}{k_{b} T}\right) I_{n}\left(h_{0}\right),
$$

где $I_{n}\left(h_{0}\right)=h_{0} \int_{0}^{t_{n}} \frac{t^{h_{0}-1}}{t+1} d t, t_{n}=\exp \left(\frac{s_{e n}}{k_{b} T h_{0}}\right), s_{e n}=\varphi_{m}-\chi_{d}-$ $-\sqrt{k e^{3} E_{f}}+\varepsilon_{e n}, \quad \varepsilon_{e n}=e \varphi\left(H_{f}\right)-\varphi_{m}-n \Delta \varepsilon$.

$\mathrm{He}$ вычисляющийся аналитически интеграл $I_{n}\left(h_{0}\right)$ можно представить в виде сходящегося ряда, удобного для проведения численных расчетов:

$$
I_{n}\left(h_{0}\right)=\left\{\begin{array}{c}
\exp \left(\frac{s_{\text {en }}}{k_{b} T}\right) \sum_{i=0}^{\infty} \frac{(-1)^{i} h_{0}}{h_{0}+i} \\
\quad \times \exp \left(\frac{s_{\text {en }}}{k_{b} T h_{0}} i\right) \text { при } s_{\text {en }} \leq 0, \\
g\left(h_{0}\right)-\exp \left(\frac{s_{\text {en }}}{k_{b} T}\right) \sum_{i=0}^{\infty} \frac{(-1)^{i} h_{0}}{\left(i+1-h_{0}\right)} \\
\quad \times \exp \left(-\frac{s_{\text {en }}}{k_{b} T h_{0}}(i+1)\right) \text { при } s_{\text {en }}>0 .
\end{array}\right.
$$

Таким образом, эмиссионная эффективность пленки, равная доле эмитированных в пленку электронов, которые выходят из нее в разряд, равна

$$
\delta_{f}=\frac{j_{e}\left(H_{f}\right)}{j_{t}\left(H_{f}\right)}=\frac{1}{g\left(h_{0}\right)} \exp \left(-\frac{H_{f}}{\lambda_{e}}\right) \sum_{n=0}^{\infty} \frac{H_{f}^{n}}{n ! \lambda_{e}^{n}} I_{n}\left(h_{0}\right) .
$$

В предельном случае $e \varphi\left(H_{f}\right) / k_{b} T \rightarrow \infty$ из полученных выражений следует, что $t_{n} \rightarrow \infty$ и $I\left(h_{0}\right) \rightarrow g\left(h_{0}\right)$, а следовательно $\delta_{f} \rightarrow 1$, поскольку при достаточно большом падении напряжения на пленке все эмитируемые в нее из металла электроны имеют у ее внешней границы энергии, достаточные для выхода в разряд. Если же считать электронную эмиссию чисто термической, т.е. происходящей над потенциальным барьером, то нужно перейти к пределу $h_{0} \rightarrow 0$, что дает $g\left(h_{0}\right)=1$ и $I\left(h_{0}\right)=\exp \left(s_{e n} / k_{b} T\right)$. В результате из (4) следует формула Ричардсона-Дэшмана, в которой учитывается снижение высоты потенциального барьера в электрическом поле (эффект Шоттки) $[6,7,11]$, а соотношение (10) для эмиссионной эффективности пленки принимает вид:

$$
\delta_{f}=\exp \left(-\frac{H_{f}}{\lambda_{e}}\right) \sum_{n=0}^{\infty} \frac{H_{f}^{n}}{n ! \lambda_{e}^{n}} \exp \left(\frac{s_{e n}}{k_{b} T}\right),
$$

где, если величина $s_{e n}$ получается положительной, нужно использовать значение $s_{e n}=0$, так как при рассматриваемых условиях электроны, испытавшие $n$ столкновений при движении в пленке, имеют энергию, достаточную для выхода из нее в разряд.

Накопление поверхностного заряда на пленке прекращается и все параметры разряда перестают зависеть от времени, когда напряженность электрического поля $E_{f}$ в пленке достигает значения, при котором доля электронов, эмитируемых из металлической подложки катода в пленку, которые нейтрализуют поверхностный заряд, становится равной доле ионного тока $\alpha$ в разрядном токе у поверхности катода, т. е. выполняется условие

$$
1-\delta_{f}\left(E_{f}\right)=\alpha
$$


При этом плотность тока термополевой эмиссии из подложки в диэлектрическую пленку в соответствии с (6) должна совпадать с плотностью разрядного тока $j$ у поверхности катода, т. е.

$$
j_{t}\left(H_{f}\right)=j .
$$

Подстановка в (12) и (13) выражений (4), (6), (7), (9) и (10) дает систему уравнений для нахождения температуры катода $T$ и напряженности электрического поля $E_{f}$ в пленке при заданных значениях $j$ и $\alpha$.

Для самосогласованного же нахождения характеристик разряда нужно дополнить эту систему уравнениями катодного слоя дугового разряда и уравнением теплопроводности в объеме катода $[4,8,11,32,33]$.

\section{Результаты численных расчетов}

Вычисления проводились для вольфрамового катода с диэлектрической пленкой оксида алюминия толщиной $H_{f}=10 \mathrm{~nm}$ на его поверхности при $\alpha=0.3$ [32,34], так как для оксида алюминия известны значения всех необходимых параметров $[11,25,26,28]: m_{e}^{*}=m_{e}$, $\varphi_{m}=4.5 \mathrm{eV}, \chi_{d}=2 \mathrm{eV}, \Delta \varepsilon=0.125 \mathrm{eV}, \lambda_{e}=0.3 \mathrm{~nm}$.

На рис. 2 приведена зависимость эмиссионной эффективности пленки $\delta_{f}$ от напряженности электрического поля $E_{f}$ в ней, найденная при $T=1500 \mathrm{~K}$, с учетом и без учета усиления термической эмиссии из металлической подложки электрическим полем, т.е. из соотношений (10) и (11). Из него видно, что при $E_{f}>5 \cdot 10^{8} \mathrm{Vm}^{-1}$ заметная доля $\delta_{f}$ электронов, эмитированных из подложки катода в пленку, имеет у ее внешней поверхности энергии, достаточные для выхода в разрядный объем, и вносит вклад в эмиссионный ток с катода. Значение $\delta_{f}$ быстро возрастает при увеличении $E_{f}$ и при $E_{f}>1.0 \cdot 10^{9} \mathrm{Vm}^{-1}$ достигает максимальной величины $\delta_{f}=1$, поскольку при такой напряженности электрического поля все электроны приобретают в пленке энергию, достаточную для выхода из нее. При этом $\delta_{f}$ принимает значение $1-\alpha=0.7$, обеспечивающее выполнение соотношения (12), т.е. достижение стационарного режима разряда при $E_{f} \approx 7 \cdot 10^{8} \mathrm{Vm}^{-1}$.

Электроны, туннелирующие в пленку через верхнюю часть потенциального барьера, имеют в металле, а следовательно, и в пленке меньшие энергии, чем электроны, эмитируемые поверх барьера, что обусловливает небольшое снижение эмиссионной эффективности пленки при учете усиления термической эмиссии электрическим полем. Однако, как следует из рис. 3, где изображена зависимость от $E_{f}$ плотности тока, эмитируемого с поверхности катода, его величина при учете усиления полем термической эмиссии может в несколько раз превосходить значение, найденное без учета этого фактора.

На рис. 4 представлена рассчитанная из (12),(13) зависимость температуры поверхности катода в разряде от плотности разрядного тока. Из него следует, что при рассматриваемых условиях температура вольфрамового

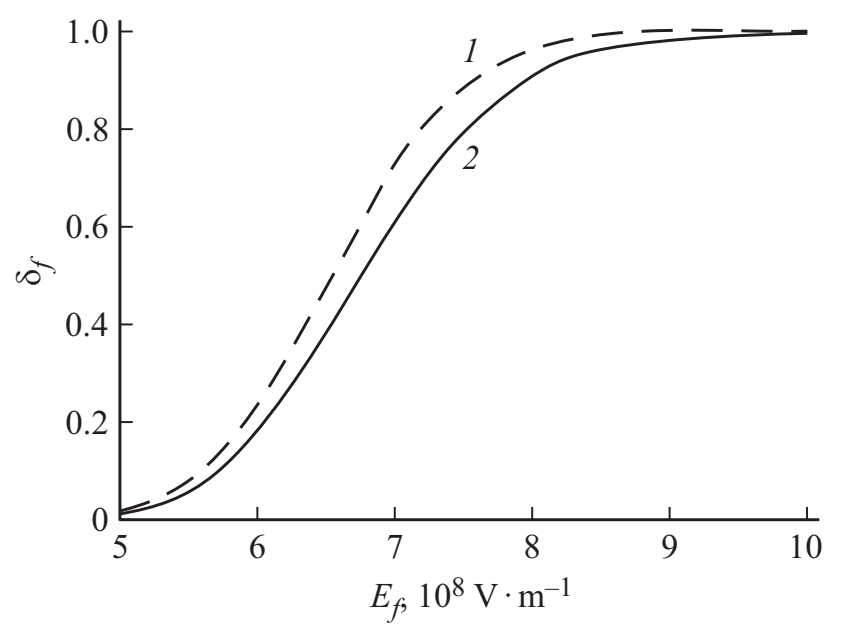

Рис. 2. Зависимость эмиссионной эффективности пленки на катоде от напряженности электрического поля в ней без учета (1) и при учете (2) усиления термической электронной эмиссии.

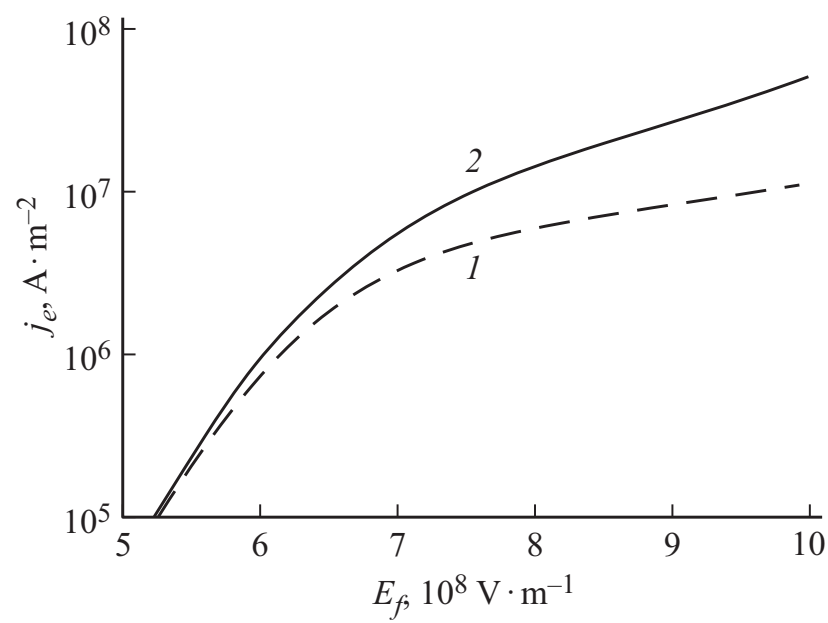

Рис. 3. Зависимость плотности эмиссионного тока с катода от напряженности электрического поля в диэлектрической пленке. Обозначения те же, что на рис. 2.

катода с диэлектрической пленкой равна $1400-1700 \mathrm{~K}$ (что согласуется с результатами измерений для ртутных ламп $[35,36])$, в то время как для такого же катода без диэлектрической пленки она имеет величину около $3000 \mathrm{~K}[8,11,32]$. Это объясняется тем, что, как следует из рис. 1 , при термической эмиссии электронов из металлического катода в разрядный объем высота потенциального барьера на его поверхности равна $\varphi_{m}$, в то время как при их эмиссии из металлической подложки катода в пленку высота барьера равна $\varphi_{m}-\chi_{d}$, т.е. является существенно меньшей. В процессе же движения эмитированных в пленку электронов они ускоряются в ней электрическим полем, что при выполнении условия (12) позволяет части из них преодолеть барьер на границе пленки и выйти в разрядный объем, создавая необходимый эмиссионный ток с катода. 


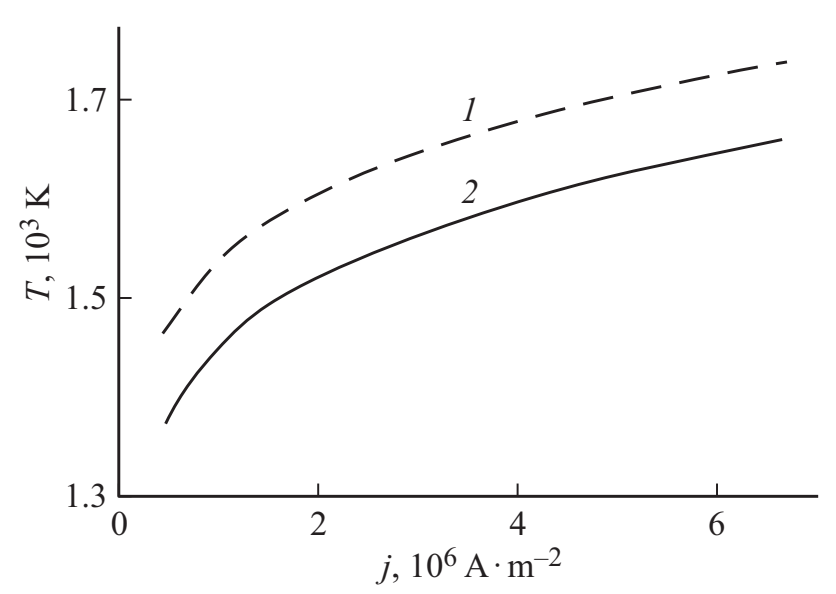

Pис. 4. Зависимость температуры поверхности катода в разряде от плотности разрядного тока. Обозначения те же, что на рис. 2.

Из рис. 4 видно так же, что усиление термической эмиссии электрическим полем позволяет обеспечить заданную плотность разрядного тока при температуре катода, примерно на $100 \mathrm{~K}$ более низкой, чем в случае чисто термической эмиссии.

\section{Заключение}

В работе предложена модель усиленной электрическим полем термической эмиссии электронов из металлической подложки катода в тонкую диэлектрическую пленку на его поверхности. Получено аналитическое выражение для эмиссионной эффективности пленки в дуговом разряде, равной доле таких электронов, которая выходит из пленки в разряд и создает эмиссионный ток с катода. Сформулирована система уравнений для температуры поверхности катода в разряде и напряженности электрического поля в пленке, обеспечивающих необходимую плотность разрядного тока.

Рассчитана зависимость эмиссионной эффективности диэлектрической пленки и плотности эмиссионного электронного тока у поверхности катода от напряженности электрического поля в ней. Показано, что наличие диэлектрической пленки может приводить к существенному снижению температуры катода в разряде вследствие меньшей высоты потенциального барьера на границе металл-диэлектрик, чем на границе металлразряд при ее отсутствии.

Установлено, что учет усиления термической эмиссии электрическим полем в разряде с катодом, на поверхности которого имеется тонкая диэлектрическая пленка, приводит к дополнительному уменьшению температуры катода при той же плотности разрядного тока на величину порядка $100 \mathrm{~K}$, и этот механизм нужно принимать во внимание при моделировании дугового разряда в случае наличия на катоде тонкой диэлектрической пленки.

\section{Финансирование работы}

Работа выполнена в рамках Программы фундаментальных исследований Научно-исследовательского университета „Высшая школа экономики“, а также в рамках реализации государственного задания „Организация проведения научных исследований“ Министерства науки и высшего образования РФ в Московском государственном техническом университете им. Н.Э. Баумана (национальном исследовательском университете) (проект 3.8408.2017/6.7) при финансовой поддержке Российского фонда фундаментальных исследований и Правительства Калужской области (проект № 18-42-400001).

\section{Конфликт интересов}

Авторы заявляют, что у них нет конфликта интересов.

\section{Список литературы}

[1] Райзер Ю.П. Физика газового разряда. Долгопрудный: ИД Интеллект, 2009. 736 с. [Raizer Y.P. Gas Discharge Physics. Berlin, Springer: 1991. 449 p.]

[2] Кудрявщев А.А., Смирнов А.С., Цендин Л.Д. Физика тлеющего разряда. СПб.: Лань, 2010. 512 с.

[3] Lister G.G., Lawler J.E., Lapatovich W.P., Godyak V.A. // Rev. Mod. Phys. 2004. Vol. 76. N 2. P. 541-598.

DOI: 10.1103/RevModPhys.76.541

[4] Сайбутдинов А.И., Файрушин И.И., Кашапов Н.Ф. // Письма в ЖЭТФ. 2016. Т. 104. Вып. 3. С. 178-183. DOI: $10.7868 / \mathrm{S} 0370274 \mathrm{X} 16150078$

[5] Бондаренко Г.Г., Фишер М.Р., Кристя В.И. // ЖТФ. 2017. т. 87. Вып. 2. С. 197-203.

DOI: 10.21883/JTF.2017.02.44125.1918 [Bondarenko G.G., Fisher M.R., Kristya V.I. // Tech. Phys. 2017. Vol. 62. N 2. P. 223-229. DOI: 10.1134/S1063784217020050]

[6] Murphy E.L., Good R.H. // Phys. Rev. 1956. Vol. 102. N 6. P. 1464-1473. DOI: 10.1103/PhysRev.102.1464

[7] Modinos A. Field, Thermionic, and Secondary Electron Emission Spectroscopy. NY.: Plenum Press, 1984. 375 p.

[8] Lichtenberg S., Nandelstädt D., Darbinghausen L., Redwitz M., Luhmann J., Mentel J. // J. Phys. D: Appl. Phys. 2002. Vol. 35. N 14. P. $1648-1656$. DOI: $10.1088 / 0022-3727 / 35 / 14 / 305$

[9] Птицын В.Э. // ЖТФ. 2007. Т. 77. Вып. 4. С. 113-118. [Ptitsin V. E. // Tech. Phys. 2007. Vol. 52. N 4. P. 504-509. DOI: $10.1134 / \mathrm{S} 1063784207040172]$

[10] Ptitsin V.E. // J. Phys. Conf. Ser. 2011. Vol. 291. N 1. P. 012019. DOI: 10.1088/1742-6596/291/1/012019

[11] Benilov M.S., Benilova L.G. // J. Appl. Phys. 2013. Vol. 114. N 6. P. 063307. DOI: $10.1063 / 1.4818325$

[12] Venkattraman A. // Appl. Phys. Lett. 2014. Vol. 104. N 19. P. 194101. DOI: $10.1063 / 1.4875237$

[13] Haase J.R., Go D.B. // J. Phys. D. 2016. Vol. 49. N 5. P. 055206. DOI: 10.1209/0295-5075/120/25002

[14] Benilov M.S., Marotta A. // J. Phys. D. 1995. Vol. 28. N 9. P. $1869-1882$. DOI: $10.1088 / 0022-3727 / 28 / 9 / 015$

[15] Coulombe S., Meunier J.-L. // J. Phys. D. 1997. Vol. 30. N 20. P. 776-780. DOI: 10.1088/0022-3727/30/20/019 
[16] Harworth F.E. // Phys. Rev. 1950. Vol. 80. N 2. P. 223-226. DOI: 10.1103/PhysRev.80.223

[17] Добрецов Л.Н., Гомоюнова М.В. Эмиссионная электроника. М.: Наука, 1966. 564 с.

[18] Lutz V.F. // IEEE Tr. Plasma Sci. 1974. Vol. 2. N 1. P. 1-10. DOI: $10.1109 /$ TPS.1974.4316799

[19] Кристя В.И., Йе Наинг Тун // Изв. РАН. Сер. физ. 2014. т. 78. Вып. 6. С. 752-759. DOI: $10.7868 / \mathrm{S} 0367676514060179$ [Kristya V.I., Ye Naing Tun // Bull. Russ. Acad. Sci. Phys. 2014. Vol. 78. N 6. P. 549-552. DOI: $10.3103 / \mathrm{S} 1062873814060161]$

[20] Riedel M., Düsterhöft H., Nagel F. // Vacuum. 2001. Vol. 61. N 2. P. 169-173. DOI: $10.1016 / \mathrm{S} 0042-207 \mathrm{X}(01) 00112-9$

[21] Bondarenko G.G., Fisher M.R., Kristya V.I., Prassitski V.V. // Vacuum. 2004. Vol. 73. N 2. P. 155159. DOI: $10.1016 /$ j.vacuum.2003.12.004

[22] Hadrath S., Ehlbeck J., Lieder G., Sigeneger F. // J. Phys. D. 2005. Vol. 38. N 17. P. 3285-3295. DOI: $10.1088 / 0022-3727 / 38 / 17 / \mathrm{S} 33$

[23] Moon K.S., Lee J., Whang K.-W. // J. Appl. Phys. 1999. Vol. 86. N 7. P. 4049-4051. DOI: $10.1063 / 1.371328$

[24] Stamenković S.N., Marković V.Lj., Gocić S.R., Jovanović A.P. // Vacuum. 2013. Vol. 89. P. 62-66. DOI: 10.1016/j.vacuum.2012.09.010

[25] Suzuki M., Sagawa M., Kusunoki T., Nishimura E., Ikeda M., Tsuji K. // IEEE Tr. Electron. Dev. 2012. Vol. 59. N 8. P. 2256 2262. DOI: 10.1109/TED.2012.2197625

[26] Bondarenko G.G., Kristya V.I., Savichkin D.O. // Vacuum. 2018. Vol. 149. P. 114-117. DOI: $10.1016 /$ j.vacuum.2017.12.028

[27] Eckertová L. // Int. J. Electron. 1990. Vol. 69. N 1. P. 65-78. DOI: $10.1080 / 00207219008920292$

[28] Hickmott T.W. // J. Appl. Phys. 2000. Vol. 87. N 11. P. $7903-$ 7912. DOI: $10.1063 / 1.373474$

[29] Егоров Н.В., Антонов А.Ю., Демченко Н.С. // ЖТФ. 2017. T. 87. Вып. 2. C. $175-181$. DOI: 10.21883/JTF.2017.02.44122.1826

[Egorov N.V., Antonov A.Yu., Demchenko N.S. // Tech. Phys. 2017. Vol. 62. N 2. P. 201-207.

DOI: $10.1134 / \mathrm{S} 1063784217020098]$

[30] Semet V., Adessi C., Capron R., Binh V.T. // Phys. Rev. B. 2007. Vol. 75. N 4. P. 045430. DOI: $10.1116 / 1.2436493$

[31] Savoye E.D., Anderson D.E. // J. Appl. Phys. 1967. Vol. 38. N 8. P. 3245-3265. DOI: $10.1063 / 1.1710096$

[32] Flesch P., Neiger M. // J. Phys. D. 2002. Vol. 35. N 17. P. 1681-1694. DOI: $10.1088 / 0022-3727 / 35 / 14 / 308$

[33] Кристя В.И. // Поверхность. 2009. Вып. 4. С. 38-40. [Kristya V.I. // J. Surf. Investig. 2009. Vol. 3. N 2. P. 289 291. DOI: $10.1134 / \mathrm{S} 1027451009020220]$

[34] Bötticher R., Bötticher W. // J. Phys. D. 2000. Vol. 33. N 4. P. 367-374. DOI: $10.1088 / 0022-3727 / 33 / 4 / 309$

[35] Рохлин Г.Н. Разрядные источники света. М.: Энергия, $1991.720 \mathrm{c}$.

[36] Бондаренко Г.Г., Кристя В.И., Фишер М.Р. // Изв. РАН. Сер. физ. 2006. Т. 70. Вып. 8. С. 1172-1174. 